\title{
Embeddability Problems for Upward Planar Digraphs ${ }^{\star}$
}

\author{
Francesco Giordano $^{1}$, Giuseppe Liotta ${ }^{1}$, and Sue H. Whitesides ${ }^{2}$ \\ ${ }^{1}$ Università degli Studi di Perugia, Italy \\ \{giordano, liotta\}@diei.unipg.it \\ ${ }^{2}$ McGill University, Canada \\ sue@cs.mcgill.ca
}

\begin{abstract}
We study two embedding problems for upward planar digraphs. Both problems arise in the context of drawing sequences of upward planar digraphs having the same set of vertices, where the location of each vertex is to remain the same for all the drawings of the graphs. We develop a method, based on the notion of book embedding, that gives characterization results for embeddability as well as testing and drawing algorithms.
\end{abstract}

\section{Introduction}

In the upward point-set embeddability problem with mapping the input is an upward planar digraph $G$ with $n$ vertices, a set $S$ of $n$ distinct points in the plane, and a mapping $\Phi$ from the vertices of $G$ to the points of $S$. The desired output is an upward planar drawing of $G$ with the vertices located at the points of $S$ assigned by the mapping. Not all instances of this problem admit a solution, as shown in Fig. 1(a) (there is no choice of upward direction with respect to which the location of vertex 1 is lowest).

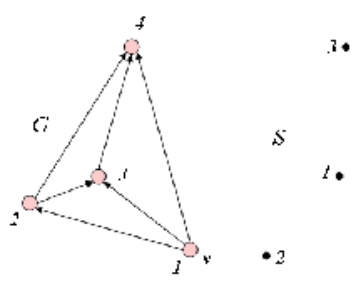

(a)
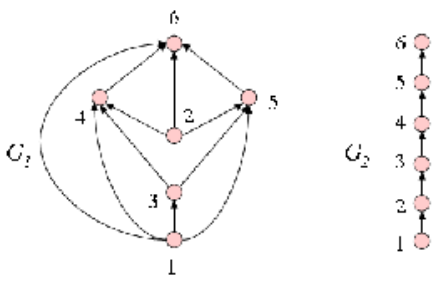

(b)

Fig. 1. (a) An upward planar digraph $G$, a set $S$ of points, and a mapping of the vertices to the points of $S$ such that an upward point-set embedding of $G$ on $S$ does not exist. (b) Two upward planar digraphs whose union is acyclic but that do not admit an upward consistent simultaneous embedding.

\footnotetext{
* This work is partially supported by the MIUR Project "MAINSTREAM: Algorithms for massive information structures and data streams", and by NSERC.
} 
In the upward consistent simultaneous embeddability problem the input is a sequence of upward planar digraphs that have the same vertex set. The desired output is a set $S$ of points in the plane and a mapping from the vertices to the points such that all the digraphs have an upward point-set embedding on $S$ with respect to a common, upward direction. Clearly, a solution exists only if the union of the digraphs is acyclic. However, this condition is not sufficient: the union of the two digraphs of Fig.[1(b) is acyclic, yet, as can be checked by straightforward case analysis, there is no simultaneous upward embedding with respect to a common direction.

Note that in the first problem, referred to as the point-set embeddability problem for short, the desired locations of the vertices are specified by $\Phi$ in the problem input, and only one graph is given. In the second problem, referred to as the simultaneous embeddability problem for short, the locations for the vertices are to be computed, and several graphs are given in the input. These problems arise in the context of computing drawings for a set or sequence of graphs under two different scenarios. In the first scenario, the graphs are specified one at a time, and the vertex locations for the drawing of the first graph determine the vertex locations for all the remaining drawings. Hence for each graph after the initial one, the locations for its drawings are specified. This gives rise to the point-set embeddability problem. An example of this scenario is provided by the visual analysis of self-modifiable code, based on computing a sequence of drawings whose edges are defined at run-time (see, e.g., [9]). In the second scenario, the graphs are all known from the outset. This gives rise to the simultaneous embeddability problem. This scenario occurs, for example, in the visual comparison of several phylogenetic trees proposed for the same organisms.

\subsection{Summary of Main Results}

Our first main result, of interest on its own, provides a tool for obtaining the others. Namely, in Sect. 3, we prove that a planar st-digraph together with any given topological numbering $\rho$ admits an upward topological book embedding such that the ordering of the vertices along the spine is $\rho$. The number of spine crossings per edge is at most $2 n-4$, which is asymptotically worst-case optimal ( $n$ is the number of vertices).

For the point-set embeddability problem, we characterize in Sect. 4 those instances that admit a solution, providing an $O\left(n^{2}\right)$-time drawing algorithm that produces at most $2 n-3$ bends per edge, which is worst-case asymptotically optimal. Then in Sect.5 we give an $O\left(n^{3}\right)$-time testing algorithm.

For the simultaneous embedding problem, in Sect. 6 we give a combinatorial characterization of instances that admit a solution.

\subsection{Related Results}

Both the embeddability problems we consider have mainly been studied for planar undirected graphs. In that case, Halton [9] proved that every instance of the point-set embeddability problem has a solution. Pach and Wenger [13] showed that solutions can 
require $\Omega(n)$ bends per edge and showed how to construct drawings with at most $O(n)$ bends per edge. See [1] for recent extensions and improvements. See Frati, Kaufmann, and Kobourov [5] for an extensive survey of simultaneous embeddability problems. Simultaneous embeddability for upward planar digraphs has been recently undertaken in [6], but for two digraphs, and without the requirement for the same choice of upward direction.

For book embeddings, see, e.g., [46] for the notion of an upward planar drawing where the vertices are aligned along a spine in a specified order, and edges are drawn as monotone curves that can cross the line. See [1] for results on book embeddings of undirected planar graphs.

For reasons of space, some proofs have been omitted and can be found in [7].

\section{Preliminaries}

We assume familiarity with basic graph drawing terminology [2]12]. A digraph is a directed graph. Let $G$ be a digraph and let $u, v$ be any two vertices of $G ;(u, v)$ denotes the directed edge from $u$ to $v$. A topological ordering of a planar digraph $G$ with $n$ vertices is a mapping $\rho$ of its vertices to distinct integers such that for every edge $(u, v)$ we have $\rho(u)<\rho(v)$. A topological numbering is a topological ordering where the vertices are mapped to integers $1, \ldots, n$. Let $u$ and $v$ be two vertices of a digraph with a given topological numbering $\rho$; if $\rho(u)<\rho(v)$ we say that $u$ precedes $v$. A topological numbering of a planar digraph with $n$ vertices can be computed in $O(n)$ time using standard graph search techniques [3].

A drawing of a digraph $G$ maps each vertex of $G$ to a distinct point in the plane and each edge $(u, v)$ of $G$ to a simple Jordan curve oriented from the point representing $u$ to the point representing $v$. A drawing of a digraph is planar if no two edges cross each other. A planar drawing $\Gamma$ of a digraph $G$ partitions the plane into topologically connected regions called the faces. The unbounded face is called the external face. A planar drawing of a digraph is upward if all of its edges are monotonically increasing in a common direction which is called the upward direction of the drawing. A digraph that admits an upward planar drawing is said to be upward planar. Let $\Gamma$ be an upward planar drawing of an upward planar digraph $G . \Gamma$ induces two linear lists of incoming and outgoing edges incident on each vertex $v$ of $G$. An upward planar embedding of an upward planar digraph $G$ is an equivalence class of upward planar drawings that induce the same two linear lists for each vertex of $G$ and define the same external face. An upward planar digraph $G$ with a given upward planar embedding is called an upward planar embedded digraph.

An $s t$-digraph is a biconnected acyclic digraph with exactly one source $s$ and exactly one sink $t$, and such that $(s, t)$ is an edge of the digraph. A planar st-digraph is an $s t$-digraph that is planar and embedded with vertices $s$ and $t$ on the boundary of the external face. A planar st-digraph is said to be maximal if all its faces are triangles, i.e. the boundary of each face has exactly three vertices and three edges. Given any planar st-digraph $G$ with $n$ vertices along with a topological ordering of its vertices, by using 
standard visit techniques, one can augment $G$ by adding edges in $O(n)$ time such that the resulting digraph has the same vertex set as $G$, is a maximal planar st-digraph, and preserves the given topological ordering. Hence from now on, we assume without loss of generality that planar st-digraphs are maximal.

Lemma 1. [3] Let $G$ be a planar acyclic digraph. $G$ is upward planar if and only if it is the spanning subgraph of a planar st-digraph.

A planar st-digraph that includes $G$ as a spanning subgraph is called an including planar st-digraph of $G$.

\section{Upward Topological Book Embeddings}

An upward topological book embedding of a planar st-digraph $G$ is an upward planar drawing $\Gamma$ of $G$ such that: (i) The vertices of $\Gamma$ lie on an oriented line called the spine of $\Gamma$; (ii) Each edge $(u, v)$ of $G$ is represented in $\Gamma$ as a sequence of semi-circles $c_{1}, c_{2}, \ldots c_{k}$ such that consecutive semi-circles lie on different half-planes and share a point along the spine, called a spine crossing of the edge. An example of an upward topological book embedding with the spine oriented according to increasing $y$ coordinate is given in Fig. 2. In the figure, edge $(5,7)$ consists of the concatenation of three semi-circles and has two spine crossings, while edge $(1,3)$ does not have spine crossings.

In this section we show that for any given topological numbering $\rho$, a planar $s t$ digraph always admits an upward topological book embedding such that a vertex $v$ precedes a vertex $w$ along the spine if and only if $\rho(v)<\rho(w)$. We call this type of drawing a $\rho$-constrained upward topological book embedding. This can be viewed as a constrained counterpart of [14].

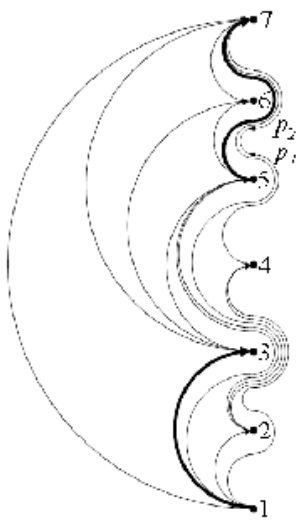

Fig. 2. An upward topological book embedding of the maximal planar st-digraph in Fig. 3(a) The vertices are ordered along the spine according to the indices of the vertices in Fig. 3(a) The drawing is computed by using the drawing algorithm of Theorem 1 


\subsection{Dual Digraph and $k$-Facial Subgraph}

Let $G$ be a maximal planar st-digraph. For each edge $e=(u, v)$ of $G$, we denote by left( $(e)$ (resp. right( $e$ )) the face to the left (resp. right) of $e$ in $G$. Let $s^{*}$ be the face $\operatorname{right}((s, t))$, and let $t^{*}$ be the face left $((s, t))$. In the rest of this section we assume that $t^{*}$ is the external face of $G$. Faces $s^{*}$ and $t^{*}$ are highlighted in Fig. 3(a) Let $G$ be a maximal planar st-digraph. The dual of $G$ is the planar st-digraph denoted as $G^{*}$ such that: (i) $G^{*}$ has a vertex for each face of $G$; (ii) $G^{*}$ has an edge $e^{*}=(\operatorname{left}(e)$, right $(e))$, for every edge $e \neq(s, t)$ of $G$; (iii) $G^{*}$ has source $s^{*}$, sink $t^{*}$, and it has edge $\left(s^{*}, t^{*}\right)$ on its external face. Figure $3(\mathrm{~b})$ depicts with dashed edges the dual digraph of the digraph of Fig. 3(a)

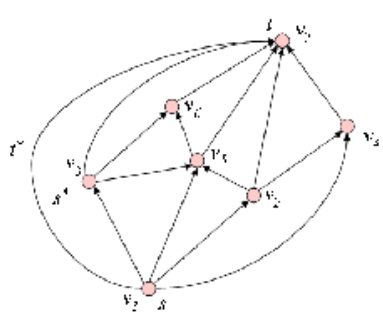

(a)

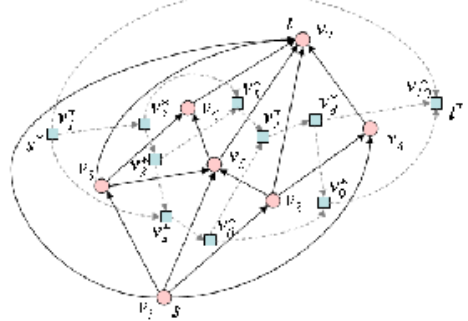

(b)

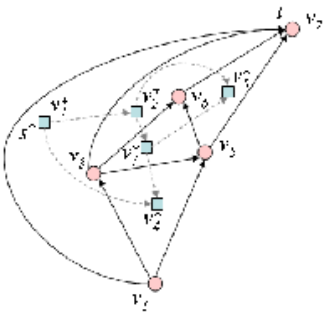

(c)

Fig. 3. (a) A planar st-digraph $G$ with a topological numbering of its vertices. (b) Planar $s t$ digraph $G$ (solid) and its dual (dashed). The vertices of the dual are numbered according to a topological numbering. (c) The 5 -facial subgraph of the maximal planar st-digraph in (a).

Property 1. Let $G$ be a maximal planar st-digraph and let $G^{*}$ be the dual digraph of $G$. Graph $G^{*}$ is a planar st-digraph (without multiple edges) with source $s^{*}$ and sink $t^{*}$.

Let $G$ be a maximal planar $s t$-digraph and let $G^{*}$ be the dual of $G$. Let $\left\{v_{1}^{*}=s^{*}, v_{2}^{*}\right.$, $\left.\ldots, v_{r}^{*}=t^{*}\right\}$ be the set of vertices of $G^{*}$ where the indices are given according to a topological numbering of $G^{*}$. See, for example, Fig. 3(b), where the vertices of the dual are numbered according to a topological numbering. By definition of dual st-digraph, a vertex $v_{i}^{*}$ of $G^{*}(1 \leq i \leq r)$ corresponds to a face of $G$; in the remainder of this section $v_{i}^{*}$ both the vertex of the dual digraph $G^{*}$ and its corresponding face in the primal digraph $G$. Let $V_{k}$ be the subset of the vertices of $G$ that belong to faces $v_{1}^{*}, v_{2}^{*}, \ldots, v_{k}^{*}$. The subgraph of $G$ induced by the vertices in $V_{k}$ is called the $k$-facial subgraph of $G$ and is denoted as $G_{k}$. Face $v_{k}^{*}$ is called the $k$-th face of $G$. Figure 3(c), for example, shows the 5-facial subgraph of the maximal planar st-digraph depicted in Figure 3(a)

Lemma 2. [6] Let $G$ be a maximal planar st-digraph with $r$ faces, let $G_{k-1}$ be the $(k-1)$-facial subgraph of $G(2 \leq k \leq r)$ and let $G_{k}$ be the $k$-facial subgraph of $G$. Let $v_{k}^{*}$ be the $k$-th face of $G$ consisting of edges $\left(w, w^{\prime}\right),\left(w^{\prime}, w^{\prime \prime}\right)$, and $\left(w, w^{\prime \prime}\right)$. One of the following statements holds. $\left(\mathbf{S}_{\mathbf{1}}\right):\left(w, w^{\prime}\right)$ and $\left(w^{\prime}, w^{\prime \prime}\right)$ are edges of the external face of $G_{k-1} ;\left(w, w^{\prime \prime}\right)$ is an edge of the external face of $G_{k} .\left(\mathbf{S}_{\mathbf{2}}\right):\left(w, w^{\prime \prime}\right)$ is an edge of the external face of $G_{k-1} ;\left(w, w^{\prime}\right)$ and $\left(w^{\prime}, w^{\prime \prime}\right)$ are edges of the external face of $G_{k}$. 
Lemma 3. [6] Let $G$ be a maximal planar st-digraph with $r$ faces and let $G_{k}$ be the $k$-facial subgraph of $G(1 \leq k \leq r) . G_{k}$ is a planar st-digraph.

\section{$3.2 \rho$-Constrained Upward Topological Book Embeddings}

Let $\Gamma$ be an upward topological book embedding of a planar st-digraph $G$, let $\Lambda$ be the spine of $\Gamma$, and let $p_{1}$ and $p_{2}$ be two vertices or two spine-crossings of $\Gamma$. Assume without loss that $\Lambda$ is vertical. The notation $p_{1}<p_{2}$ means that $p_{1}$ precedes $p_{2}$ along the spine of $\Gamma$. We denote with $\left(p_{1}, p_{2}\right)$ a semi-circle in $\Gamma$ (either an edge or a portion of an edge) with antipodal points $p_{1}$ and $p_{2}$ such that $p_{1}<p_{2}$. We say that any point $p$ of $\Lambda$ is covered if there exists a semi-circle $\left(p_{1}, p_{2}\right)$ in the half-plane on the right-hand side of $\Lambda$ such that $p_{1}<p<p_{2}$. Otherwise, we say that $p$ is visible. For example, vertex 3 of Fig. 2 is covered while vertex 4 is visible.

Let $p_{1}, p_{2}$ be two points on the spine of $\Gamma$. We say that segment $\overline{p_{1} p_{2}}$ is a maximal covered segment if every point $p$ such that $p_{1}<p<p_{2}$ is covered and there are no other segments $\overline{q_{1} q_{2}}$ with $q_{1} \leq p_{1}<p_{2} \leq q_{2}$ such that this same property holds. Similarly, $\overline{p_{1} p_{2}}$ is a maximal visible segment if all of its points are visible and it is not a subset of another visible segment. For example, segment $\overline{p_{1} p_{2}}$ in Fig. 2 is a maximal visible segment. Let $v$ be a point of $\Lambda$ that represents a vertex of $G$. We say that $v$ has an upper pocket if there exists a maximal visible segment $\overline{p_{1} p_{2}}$ such that: (i) $v \leq p_{1}<p_{2}$, (ii) no semi-circle (either in the left or in the right half-plane defined by $\Lambda$ ) has an end-point in $\overline{p_{1} p_{2}}$, and (iii) there is no vertex $u$ such that $v<u \leq p_{1}<p_{2}$. For example, segment $\overline{p_{1} p_{2}}$ in Fig. 2 is the upper pocket of vertex 5 but it is not the upper pocket of vertex 4 . Similarly the lower pocket of a vertex $v$ in $\Gamma$ is defined by considering maximal visible segments below $v$. Segment $\overline{p_{1} p_{2}}$ in Fig. 2 is the lower pocket of vertex 6 .

Theorem 1. Let $G$ be a maximal planar st-digraph and let $\rho$ be a topological numbering of $G$. $G$ admits a $\rho$-constrained upward topological book embedding with at most $2 n-4$ spine crossings per edge, which is asymptotically worst-case optimal. Also, such a $\rho$-constrained upward topological book embedding can be computed in $O\left(n^{2}\right)$ time.

Proof. We compute a $\rho$-constrained upward topological book embedding $\Gamma^{\rho}$ of $G$ by maintaining the following invariant properties: $\left(I_{1}\right)$ : Every vertex has a lower pocket and an upper pocket. $\left(I_{2}\right)$ : For every maximal covered segment $\overline{p_{1} p_{2}}$, there exists a vertex $v$ of $G$ such that $p_{1}<v<p_{2}$.

We proceed by induction on the number of internal faces of $G$.

Base Case: Refer to Fig. 4(a). Suppose $G$ has exactly one internal face $v^{*}$ and let $\{s, t, w\}$ be the vertices of the boundary of face $v^{*}$. Let $\Lambda$ be a vertical line in the plane. Draw $s$, $t$ and $w$ along $\Lambda$ such that $s<w<t$. Draw edges $(s, t),(s, w)$ and $(w, t)$ as the semicircles $(s, t),(s, w)$ and $(w, t)$ respectively, in the half-plane on the left-hand side of $\Lambda$. By construction, the resulting drawing is a $\rho$-constrained upward topological book embedding. Also, segment $\overline{s w}$ is a lower pocket for $w$ and an upper pocket for $s$, while segment $\overline{w t}$ is an upper pocket for $w$ and a lower pocket for $t$. The upper pocket for $t$ and the lower pocket for $s$ are the half lines above $t$ and below $s$, respectively. Thus Property $I_{1}$ holds. Property $I_{2}$ holds since there are no covered segments in this case. 


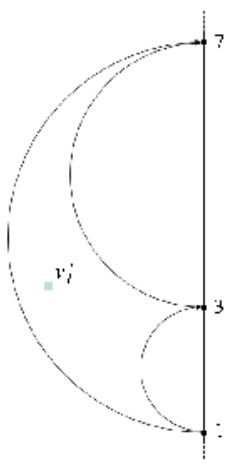

(a)

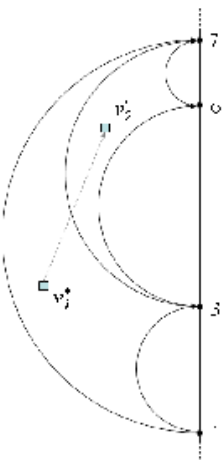

(b)

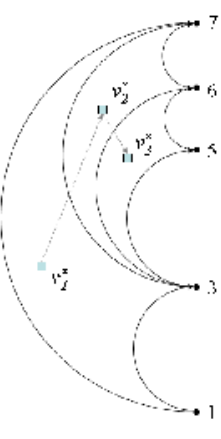

(c)

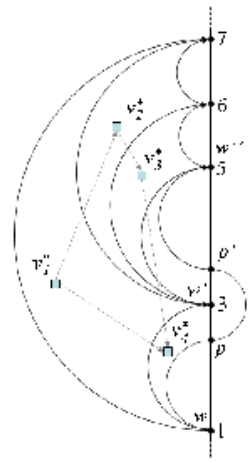

(d)

Fig. 4. Four steps of the algorithm in the proof of Theorem 1 applied to the digraph in Fig. 3(a)

Inductive case: Suppose by induction that a $\rho$-constrained upward topological book embedding of $G$ satisfying Properties $I_{1}$ and $I_{2}$ can be computed when $G$ has $k-1$ faces and assume that $G$ has $k$ faces $(k>1)$. Let $G^{*}$ be the dual of $G$ and let $\left\{v_{1}^{*}=\right.$ $\left.s^{*}, v_{2}^{*}, \ldots, v_{k}^{*}=t^{*}\right\}$ be the vertex set of $G^{*}$, where the indices are given according to a topological numbering of $G^{*}$. Also, let $G_{k-1}$ be the $(k-1)$-facial subgraph of $G$. By definition and by Lemma 3 $G_{k-1}$ is a planar st-digraph with exactly $k-1$ internal faces. By the inductive hypothesis there exists a $\rho$-constrained upward topological book embedding $\Gamma_{k-1}^{\rho}$ of $G_{k-1}$ satisfying Properties $I_{1}$ and $I_{2}$. Since $G$ has $k$ internal faces, the $k$-facial subgraph of $G$ is $G$ itself. Let $v_{k}^{*}$ be the $k$-th face of $G$ consisting of edges $\left(w, w^{\prime}\right),\left(w^{\prime}, w^{\prime \prime}\right)$, and $\left(w, w^{\prime \prime}\right)$ (see also Fig. 4). Let $\Lambda$ be the spine of $\Gamma_{k-1}^{\rho}$. We show how to compute a $\rho$-constrained upward topological book embedding $\Gamma^{\rho}$ of $G$ satisfying Properties $I_{1}$ and $I_{2}$, by adding face $v_{k}^{*}$ to $\Gamma_{k-1}^{\rho}$. We distinguish two cases depending on whether the $k$-th face of $G$ satisfies Statement $S_{1}$ or Statement $S_{2}$ of Lemma2

Statement $S_{1}$ of Lemma2 2 holds. Refer to Fig.4(d) where $v_{k}^{*}$ is face $v_{4}^{*}$ and we need to insert edge $(1,5)$. Let $j$ be the number of vertices between $w$ and $w^{\prime \prime}$ along $\Lambda$. Suppose $j=1$. Since $w, w^{\prime}$ and $w^{\prime \prime}$ are on the external face of $G_{k-1}$, by Property $I_{1}$ there are no endpoints of semi-circles on segments $\overline{w w^{\prime}}$ and $\overline{w^{\prime} w^{\prime \prime}}$. Choose two arbitrary points $p$ and $p^{\prime}$ such that $w<p<w^{\prime}<p^{\prime}<w^{\prime \prime}$. Draw edge $\left(w, w^{\prime \prime}\right)$ as three semicircles $(w, p),\left(p, p^{\prime}\right),\left(p^{\prime}, w^{\prime \prime}\right)$, respectively in the half-planes on the left hand-side, right hand-side, and left hand-side of $\Lambda$. The resulting drawing $\Gamma^{\rho}$ is a $\rho$-constrained upward topological book embedding of $G$. Also, Property $I_{1}$ holds, since $\overline{w p}$ is an upper pocket for $w$ and a lower pocket for $w^{\prime}$ and $\overline{p^{\prime} w^{\prime \prime}}$ is an upper pocket for $w^{\prime}$ and a lower pocket for $w^{\prime \prime}$. Furthermore, $\overline{p p^{\prime}}$ is a maximal covered segment and indeed $w$ is such that $p<w<p^{\prime}$. Thus, Property $I_{2}$ holds.

If the number of vertices between $w$ and $w^{\prime \prime}$ along $\Lambda$ is $j=h-1(2<h<n)$ then edge $\left(w, w^{\prime \prime}\right)$ can be added to $\Gamma_{k-1}^{\rho}$ such that the resulting drawing is a $\rho$-constrained upward topological book embedding satisfying Properties $I_{1}$ and $I_{2}$. Assume $j=h$. Let $p$ be the point of $\Lambda$ above $w$ such that $\overline{w p}$ is the upper pocket of $w$. 
Two cases are possible: (i) $p$ is the point representing vertex $w^{\prime}$; (ii) $p$ is an endpoint of a semi-circle $\left(p, p^{\prime}\right)$ in the half-plane on the right-hand side of $\Lambda$. We can deal with cases (i) and (ii) at once, since case (i) can be seen as a special instance of case (ii), where $p$ and $p^{\prime}$ coincide with vertex $w$. Thus, suppose case (ii) holds and let $\left(q, q^{\prime}\right)$ be a semi-circle of $\Gamma_{k-1}^{\rho}$ in the half plane on the right-hand side of $\Lambda$. Points $q$ and $q^{\prime}$ cannot be such that $q<p<q^{\prime}$ since every point of $\overline{w p}$ must be visible from the right-hand side, and they cannot be such that $p<q<p^{\prime}<q^{\prime}$ since in this case there would be a crossing between semi-circles $\left(q, q^{\prime}\right)$ and $\left(p, p^{\prime}\right)$. Therefore, $\overline{p p^{\prime}}$ is a maximal covered segment of $\Gamma_{k-1}^{\rho}$. Hence, by Property $I_{2}$, there must exist a vertex $v$ of $G_{k-1}$ such that $p<v<p^{\prime}$ in $\Gamma_{k-1}^{\rho}$. Also, by Property $I_{1}$, there exists a point $p^{\prime \prime}$ of $\Lambda$ such that $\overline{p^{\prime} p^{\prime \prime}}$ is the upper pocket of $v$. Let $\tilde{p}$ and $\tilde{p}^{\prime}$ be arbitrary points of $\Lambda$ such that $w<\tilde{p}<p<p^{\prime}<\tilde{p}^{\prime}<p^{\prime \prime}$.

We draw edge $\left(w, w^{\prime \prime}\right)$ by splitting it into two edges: An edge from $w$ to $\tilde{p}^{\prime}$ and an edge from $\tilde{p}^{\prime}$ to $w^{\prime \prime}$. Since there are exactly $h-1$ vertices between $\tilde{p}^{\prime}$ and $w^{\prime \prime}$, edge $\left(\tilde{p}^{\prime}, w^{\prime \prime}\right)$ can be added to $\Gamma_{k-1}^{\rho}$ so that the resulting drawing is a $\rho$-constrained upward topological book embedding satisfying Properties $I_{1}$ and $I_{2}$. Notice that, as a consequence of Property $I_{1}$, there must exist a point $p^{\prime \prime}$ with $\tilde{p}^{\prime}<p^{\prime \prime}$ such that $\overline{\tilde{p}^{\prime} p^{\prime \prime}}$ is an upper pocket for $\tilde{p}^{\prime}$, which means that the first semi-circle of edge $\left(\tilde{p}^{\prime}, w^{\prime \prime}\right)$ is in the half-plane on the left hand-side of $\Lambda$. Now, draw edge $\left(w, \tilde{p}^{\prime}\right)$ as two semi-circles $(w, \tilde{p})$ and $\left(\tilde{p}, \tilde{p}^{\prime}\right)$ in the half planes on the left-hand side and on the right-hand side of $\Lambda$, respectively. Semi-circles $(w, \tilde{p})$ and $\left(\tilde{p}, \tilde{p}^{\prime}\right)$ do not cross any other semi-circle of $\Gamma_{k-1}^{\rho}$. Also, Properties $I_{1}$ and $I_{2}$ hold for $w$ and $v$. Indeed, segment $\overline{w \tilde{p}}$ is an upper pocket for $w$ and a lower pocket for $v$ while segment $\overline{\tilde{p}^{\prime} p^{\prime \prime}}$ is an upper pocket for $v$. Property $I_{2}$ holds for $v$ as segment $\overline{\tilde{p} \tilde{p}^{\prime}}$ is a maximal covered segment and $v$ is such that $\tilde{p}<v<\tilde{p}^{\prime}$. Therefore, the semi-circles we have drawn preserve planarity and respect Properties $I_{1}$ and $I_{2}$. It follows that edge $\left(w, w^{\prime \prime}\right)$ has been drawn as a monotone curve from $w$ to $w^{\prime \prime}$ formed by a sequence of semi-circles $c_{1}, c_{2}, \ldots c_{2 h+1}$ such that consecutive semicircles lie on different half-planes, share only a spine crossing along $\Lambda$, do not cross other semi-circles and Properties $I_{1}$ and $I_{2}$ hold. The resulting drawing $\Gamma^{\rho}$ is thus a $\rho$ constrained upward topological book embedding of $G$ satisfying Properties $I_{1}$ and $I_{2}$.

Statement $S_{2}$ of Lemma 2holds. Refer also to Fig.4(b) and Fig.4(c). Let $v$ be the vertex of $G_{k-1}$ having the largest number in the topological numbering such that $\rho(v)<\rho\left(w^{\prime}\right)$. Let $\overline{p_{1} p_{2}}$ be the upper pocket of $v$. Draw vertex $w^{\prime}$ such that $p_{1}<w^{\prime}<p_{2}$. Segment $\overline{p_{1} w^{\prime}}$ is both the new upper pocket of $v$ and the lower pocket of $w^{\prime}$ while segment $\overline{w^{\prime} p_{2}}$ is the upper pocket of $w^{\prime}$. Thus, the drawing is a $\rho$-constrained upward topological book embedding satisfying Properties $I_{1}$ and $I_{2}$. Draw edge $\left(w, w^{\prime}\right)$ by the same technique as in the previous case. The same reasoning proves that the resulting drawing is a $\rho$-constrained upward topological book embedding satisfying Properties $I_{1}$ and $I_{2}$. The same argument applies to edge $\left(w^{\prime}, w^{\prime \prime}\right)$. The final drawing is thus a $\rho$-constrained upward topological book embedding of $G$ satisfying Properties $I_{1}$ and $I_{2}$.

It remains to prove the time complexity of the algorithm and the number of spine crossings per edge of the drawing. The dual digraph of $G$ and a topological numbering of its vertices can be computed in linear time. Indeed, by Property 1 the dual of $G$ is a planar st-digraph without multiple edges. Also, each edge $(u, v)$ is drawn by the algorithm so that for every vertex $w$ with $u<w<v$ there are exactly two spine 
crossings $p_{1}$ and $p_{2}$ with $p_{1}<w<p_{2}$. It follows that the number of spine crossings per edge is at most $2(n-2)=2 n-4$; we also remark that $\Omega(n)$ bends are known to be necessary for constructing topological book embeddings with a fixed ordering of undirected planar graphs [1]. Finally, the time complexity of the described drawing algorithm is $O(m \cdot n)$ that is equal to $O\left(n^{2}\right)$ since $G$ is a planar graph.

\section{Upward Point-Set Embeddability with a Given Mapping $\Phi$}

We first study the special case that the points of $S$ are collinear and that the input graph is a planar st-digraph, and then study general upward planar digraphs and points in general position.

\subsection{Collinear Points and Planar st-Digraphs}

Assume without loss that $p_{1}, \ldots, p_{n}$ are vertically aligned. We associate each point $p_{i}$ of $S$ with an integer in the set $\{1, \ldots, n\}$ such that point $p_{i}$ is given integer $k$ if $p_{i}$ is the $k$-th point of $S$ that we encounter moving along the increasing $y$-direction. We also consistently assign numbers to the vertices $v_{1}, \ldots, v_{n}$ : If point $p_{i}=\Phi\left(v_{j}\right)$ has been given integer $k$, then also $v_{j}$ is given integer $k$. See Fig. 5(a) for an example. We call such a numbering of the vertices of $G$ the $\Phi$-numbering of $G$ and we call $\Phi$-number of $v_{j}$ the number assigned to vertex $v_{j}$. We say that mapping $\Phi$ induces a topological numbering of $G$ if the $\Phi$-numbering of $G$ is also a topological numbering of $G$. For example, the $\Phi$-numbering of Fig. 5(a) does not induce a topological numbering of $G$.

The characterization almost immediately follows from the result in Sect. 3 concerning $\rho$-constrained upward topological embeddability and from the observation that the $y$-coordinates of the vertices in an upward planar drawing induce a topological numbering of the graph.

Lemma 4. Let $G$ be an upward planar digraph with $n$ vertices, $S=\left\{p_{1}, \ldots, p_{n}\right\}$ a set of vertically aligned points and $\Phi$ a mapping from $G$ to $S$. Let $\Gamma$ be an upward topological book embedding of $G$ such that: (i) the maximum number of spine crossings per edge of $\Gamma$ is $k$; (ii) for every pair of vertices $u$ and $v$ of $G$ such that $u<v$ along the spine of $\Gamma$, the $\Phi$-number of $u$ is smaller than the $\Phi$-number of $v$. Then $G$ admits an upward point-set embedding on $S$ consistent with $\Phi$, with at most $k+1$ bends per edge.

Theorem 2. Let $G$ be a planar st-digraph with $n$ vertices; let $S$ be a set of $n$ distinct collinear points in the plane; let $\Phi$ be a mapping from $G$ to $S$. $G$ admits an upward point-set embedding on $S$ consistent with $\Phi$ if only if $\Phi$ induces a topological numbering of $G$. Also, such an upward point-set embedding of $G$ on $S$ can be computed in $O\left(n^{2}\right)$ time with at most $2 n-3$ bends per edge, which is asymptotically worst-case optimal.

\subsection{Points in General Position and Upward Planar Digraphs}

In this section we extend Theorem 2 by characterizing when mapping $\Phi$ guarantees the upward point-set embeddability of a (not necessarily $s t$-) upward planar digraph $G$ 


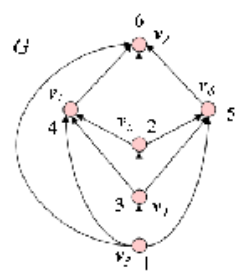

(a)

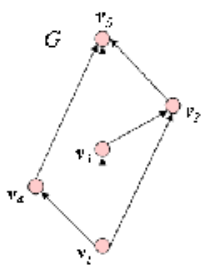

(b)

Fig. 5. (a) A planar st-digraph $G$, a set $S$ of distinct collinear points in the plane and the $\Phi$ numbering of $G$. (b) A digraph $G$ and a set $S$ of points in the plane. Mapping $\Phi$ induces a topological numbering of $G$ on $\ell$, whereas it does not induce a topological numbering of $G$ on $\ell^{\prime}$.

on a set $S$ of (not necessarily collinear) points. Let $\ell$ be a directed line. We denote as $S_{\ell}=\left\{p_{1}^{\prime}, \ldots, p_{n}^{\prime}\right\}$ the collinear set of points obtained by orthogonally projecting $S$ onto $\ell$; we assume that the direction of $\ell$ is such that when projecting $S$ on $\ell$ no two projected points coincide. Also, let $\Phi_{\ell}$ be the mapping from $G$ to $S_{\ell}$ that associates each vertex $v$ of $G$ with the projection of $\Phi(v)$ on $\ell$. We say that mapping $\Phi$ induces a topological numbering of $G$ on $\ell$ if mapping $\Phi_{\ell}$ induces a topological numbering of $G$. For example, Fig. 5(b) shows the digraph $G$, the set $S$ and the mapping $\Phi$ from $G$ to $S$ defined by associating every vertex of $G$ with the point of $S$ having the same index; also, two directed lines $\ell$ and $\ell^{\prime}$ are depicted such that $\Phi$ induces a topological numbering on $\ell$ while it does not induce a topological numbering on $\ell^{\prime}$.

Theorem 3. Let $G$ be an upward planar digraph with $n$ vertices, $S$ a set of $n$ distinct points in the plane, and $\Phi$ a mapping from $G$ to $S$. $G$ admits an upward point-set embedding consistent with $\Phi$ if and only if there exists an including planar st-digraph $G^{\prime}$ of $G$ and a directed line $\ell$ such that $\Phi$ induces a topological numbering of $G^{\prime}$ on $\ell$. Also, such an upward point-set embedding of $G$ on $S$ can be computed in $O\left(n^{2}\right)$ time with at most $2 n-3$ bends per edge, which is asymptotically worst-case optimal.

We remark that the number of bends per edge stated in Theorem 3 improves by a constant factor the best known upper bound of $3 n+2$ for the point-set embeddability with mapping of undirected planar graphs (Theorem 4 of [1]).

\section{Testing Upward Point-Set Embeddability}

Theorem 3 naturally raises the question about how to efficiently test whether an upward planar digraph $G$ with $n$ vertices admits an upward point-set embedding consistent with a given mapping $\Phi$ on a set $S$ of $n$ distinct points. By Theorem 3 , it suffices to test whether there exist an including planar st-digraph $G^{\prime}$ of $G$ and a directed line $\ell$ such 
that $\Phi$ induces a topological numbering of $G^{\prime}$ on $\ell$. Therefore, we consider every directed line $\ell$ such that $\Phi$ induces a distinct $\Phi_{\ell}$-numbering. For each such $\Phi_{\ell}$-numbering, we check whether it is a topological numbering of $G$ and, if so, we verify whether there exists an including planar st-digraph of $G$ that preserves it. The following lemma strongly relies on results by [1011] concerning level planarity testing and embedding; its proof is omitted from this abstract.

Lemma 5. Let $\rho$ be a $\Phi_{\ell}$-numbering. There exists an $O(n)$-time algorithm that tests whether $\rho$ is a topological numbering of $G$ and, if so, whether there exists an including planar st-digraph of $G$ that preserves $\rho$.

To compute all possible $\Phi_{\ell}$-numberings we must consider all possible directed lines such that the orthogonal projections of the points on these lines produce different permutations of the points. This is equivalent to computing the well-known circular sequence of permutations associated with point set $S$ (see, e.g. [8]).

Lemma 6. [8] Let $S$ be a set of $n$ distinct points in the plane. The circular sequence of permutations associated with $S$ has cardinality $O\left(n^{2}\right)$ and can be computed in $O\left(n^{2}\right)$ time.

Theorem 4. Let $G$ be an upward planar digraph, $S=\left\{p_{1}, \ldots, p_{n}\right\}$ a set of $n$ distinct points in the plane, and $\Phi$ a mapping from $G$ to $S$. There exists an $O\left(n^{3}\right)$-time algorithm that tests whether $G$ admits an upward point-set embedding on $S$ consistent with $\Phi$.

\section{Upward Consistent Simultaneous Embeddability}

The following theorem characterizes the upward simultaneous embeddability of a sequence of upward planar digraphs with respect to the same direction.

Theorem 5. A sequence $G_{1}, G_{2}, \ldots, G_{k}$ of distinct upward planar digraphs sharing the same vertex set admits an upward consistent simultaneous embedding with respect to the same direction if and only if there exists a sequence $G_{1}^{\prime}, G_{2}^{\prime}, \ldots, G_{k}^{\prime}$ such that: (i) $G_{i}^{\prime}$ is an including planar st-digraph of $G_{i}$, and $(i i) \bigcup_{i=1}^{k} G_{i}^{\prime}$ is acyclic.

Proof. If the sequence $G_{1}, G_{2}, \ldots, G_{k}$ admits the desired embedding, then each drawing is an upward planar drawing of $G_{i}(1 \leq i \leq k)$ and has an including planar stdigraph by Lemma 1 Since all drawings have the same direction of upwardness, the union of these planar st-digraphs is acyclic.

Let $n$ be the number of vertices in each graph of the sequence and let $G^{\prime}$ be the union digraph, that is $G^{\prime}=\bigcup_{i=1}^{k} G_{i}^{\prime}$. Assume that $G^{\prime}$ is acyclic and let $\rho$ be a topological numbering of $G^{\prime}$. Note that $\rho$ is a topological numbering of each $G_{i}^{\prime}$. Compute a $\rho$-constrained upward topological book embedding for each $G_{i}^{\prime}$ by using Theorem 1 Define a set of $n$ distinct points in the plane having consecutive $y$-coordinates from 1 to $n$. Define a mapping $\Phi$ that associates every vertex of $G_{i}^{\prime}$ having number $h$ in the topological numbering with the point of $S$ having $y$-coordinate equal to $h$. By Theorem 3 each $G_{i}^{\prime}$ has an upward point-set embedding consistent with $\Phi$ such that the edges are monotonically increasing with the $y$-direction. 


\section{Open Problems}

We conclude with three open problems: (1) For upward point-set embedding with a given mapping, minimize the total number of bends; (2) Improve the time complexity in Theorem 4, and (3) Design a fast test for upward simultaneous embeddability with respect to the same direction (we have linear time results for the case of switch-regular digraphs).

\section{References}

1. Badent, M., Di Giacomo, E., Liotta, G.: Drawing colored graphs on colored points. In: Dehne, F., Sack, J.-R., Zeh, N. (eds.) WADS 2007. LNCS, vol. 4619, pp. 102-113. Springer, Heidelberg (2007)

2. Di Battista, G., Eades, P., Tamassia, R., Tollis, I.: Graph Drawing. Prentice-Hall, Englewood Cliffs (1999)

3. Di Battista, G., Tamassia, R.: Algorithms for plane representations of acyclic digraphs. Theoretical Computer Science 61(2-3), 175-198 (1988)

4. Enomoto, H., Miyauchi, M.: Embedding graphs into a three page book with $O(M \log N)$ crossings of edges over the spine. SIAM J. of Discrete Math. 12(3), 337-341 (1999)

5. Frati, F., Kaufmann, M., Kobourov, S.: Constrained simultaneous and near-simultaneous embeddings. In: Hong, S.-H., Nishizeki, T., Quan, W. (eds.) GD 2007. LNCS, vol. 4875, pp. 268-279. Springer, Heidelberg (2008)

6. Giordano, F., Liotta, G., Mchedlidze, T., Symvonis, A.: Computing upward topological book embeddings of upward planar digraphs. In: Tokuyama, T. (ed.) ISAAC 2007. LNCS, vol. 4835, pp. 172-183. Springer, Heidelberg (2007)

7. Giordano, F., Liotta, G., Whitesides, S.: Drawing a sequence of upward planar digraphs: Characterization results and testing algorithms. Tech. rep., Università degli Studi di Perugia, RT-008-01 (2008)

8. Goodman, J.E., Pollack, R.: On the combinatorial classification of nondegenerate configurations in the plane. J. of Combinatorial Theory, Ser. A 29(2), 220-235 (1980)

9. Halton, J.H.: On the thickness of graphs of given degree. Inform. Sciences 54, 219-238 (1991)

10. Jünger, M., Leipert, S.: Level planar embedding in linear time. J. of Graph Algorithms and Applications 6(1), 67-113 (2002)

11. Jünger, M., Leipert, S., Mutzel, P.: Level planarity testing in linear time. In: Whitesides, S.H. (ed.) GD 1998. LNCS, vol. 1547, pp. 224-237. Springer, Heidelberg (1999)

12. Kaufmann, M., Wagner, D. (eds.): Drawing Graphs. LNCS, vol. 2025. Springer, Heidelberg (2001)

13. Pach, J., Wenger, R.: Embedding planar graphs at fixed vertex locations. Graphs and Combin. 17(4), 717-728 (2001) 